\section{RMD Open}

Rheumatic \&

Musculoskeletal Diseases

\title{
Development of a patient decision aid for the initiation of urate-lowering therapy in gout patients
}

\section{Ritch te Kan
Hein de}

Boonen A, Jansen T, et al. Development of a patient decision aid for the initiation of urate-lowering therapy in gout patients. RMD Open 2022;8:e001979. doi:10.1136/ rmdopen-2021-001979

- Additional supplemental material is published online only. To view, please visit the journal online (http://dx.doi.org/10. 1136/rmdopen-2021-001979).

Received 27 September 2021 Accepted 23 January 2022

Check for updates

(C) Author(s) (or their employer(s)) 2022. Re-use permitted under CC BY-NC. No commercial re-use. See rights and permissions. Published by BMJ.

${ }^{1}$ Internal Medicine, Division of Rheumatology, Maastricht University Medical Centre+, Maastricht, Netherlands ${ }^{2}$ Care and Public Health Research Institute (CAPHRI), Maastricht University, Maastricht, Netherlands ${ }^{3}$ Rheumatology, VieCuri Medical Centre, Venlo, Netherlands ${ }^{4}$ Health Promotion, Maastricht University, Maastricht, Limburg, Netherlands

${ }^{5}$ Rheumatology, Centre Hospitalier Chrétien, Liege, Belgium

Correspondence to Dr Ritch te Kampe; r.tekampe@maastrichtuniversity. $\mathrm{nl}$ preferred ULT.
Aim Shared decision-making improves patients' experiences with care, satisfaction with management decisions and possibly health outcomes. This study describes the development of a decision aid (DA) that supports patients with gout and their physicians in a faceto-face clinical setting to (a) decide whether or not to ( $r$ ) start urate-lowering therapy (ULT) and (b) agree on the

Methods Recommendations of the International Patient Decision Aid Standards group guided the development. A steering group of experts in gout and health services research specified the scope. Nominal group technique meetings were organised in which patients ranked the importance of preidentified potential characteristics/ attributes of ULT and discussed further needs regarding the DA. A literature search was conducted to collect evidence on gout outcomes with and without ULT. Subsequently, the DA prototype was designed and adjusted using feedback from the steering group and results of cognitive debriefing interviews among five gout patients.

Results The final DA consists of six pages. First, the DA clarifies the decision at stake and describes gout including its risk factors, the role of lifestyle and treatment of flares. Next, risk of future flares with and without ULT in relation to serum uric acid levels is described and visualised. Relevant attributes of ULT are presented in an option grid distinguishing first-line and second-line ULT. Finally, patients' believes and preferences are explicitly addressed before making the shared decision.

Conclusion This study provides initial support for usability of a DA for gout patients eligible for starting ULT.

\section{INTRODUCTION}

Gout is worldwide the most common form of inflammatory arthritis and is a well treatable disease. ${ }^{1}$ Serum uric acid (sUA) is the main risk factor for gout. Lifestyle modifications, especially weight loss in case of obesity, play a relevant but limited role in controlling gout. ${ }^{23}$ Therefore, most patients will require pharmacological urate-lowering therapy (ULT) to prevent recurrent gout flares and damage related to tophi, and possibly to reduce risk for comorbidities. ${ }^{4}$

\section{Key messages}

What is already known about this subject?

- Management recommendations for gout state that patients should be fully involved in decision-making concerning the initiation of urate-lowering therapy after a first gout flare.

What does this study add?

- We developed a decision aid to support patients and physicians with the decision to (re-)start uratelowering therapy for patients with gout.

- The decision aid is based on evidence from the literature, views of experts and perspectives of gout patients.

How might this impact on clinical practice or future developments?

- The decision aid can optimise communication, confidence with treatment decisions and possibly medication adherence among gout patients.

Several ULTs are available to reduce sUA. Allopurinol and febuxostat inhibit the activity of xanthine oxidase and, thus, reduce uric acid production. Benzbromaron and lesinurad are examples of uricosuric drugs and increase the renal excretion of sUA. ${ }^{56}$ Despite the availability of an increasing number and mode of actions of ULT, gout management is far from optimal. ${ }^{7-10}$ Suboptimal treatment is related to various key barriers among both physicians and patients. ${ }^{11-13}$ Importantly, a qualitative study revealed that a substantial proportion of patients receives contradictory information from different physician, contributing to poor treatment initiation and adherence. $^{14}$

Several initiatives have been proposed to improve outcomes of gout treatment in daily practice. $^{15}$ Shared decision-making (SDM) is increasingly considered to constitute an essential part of quality of care and is grounded in the paradigm that care should 
be based on best evidence and should be respectful of, and responsive to, individual patient preferences, needs and values. ${ }^{16}$ Decision aids (DAs) are tools that support patients and physicians in the choices when decisions about screening, treatment or other interventions have to be made. ${ }^{17-19}$ Shared decisions involve at minimum a patient and physician, although other healthcare providers or friends and family members may be invited to participate. ${ }^{2021}$ The process ensures that correct and complete information is readily available for patients and physicians. ${ }^{22}$ While effectiveness of DAs on disease outcomes is as yet contradictory, patients exposed to DAs feel more knowledgeable, better informed and more clear about their values. ${ }^{22}$

The European Alliance of Associations for Rheumatology (EULAR) and the American College of Rheumatology (ACR) recommends to initiate ULT after a first gout flare. ${ }^{23} 24$ EULAR recommendation specifically stated that: 'Patients with gout should receive full information and be fully involved in decision-making concerning the use of ULT to increase uptake and adherence of ULT, ${ }^{23}$ To support the implementation of this recommendation and, thus, improve quality of care, this paper describes the development of a DA for patients with gout that have an indication to (re-)start ULT in a clinical setting.

\section{METHODS}

The study protocol was determined within a steering group consisting of four rheumatologists and two health service researchers including an expert on DAs. Recommendations by the International Patient Decision Aid Standards (IPDAS) group guided the development and comprised four phases: scoping, design (patients and physicians needs assessment and literature searches), development of a prototype and pilot testing with patients. ${ }^{18}{ }^{25}$ The study was approved by the ethical committee of Maastricht University Medical Center (2018-0801).

\section{Phase 1: scope}

The aim was to develop a DA to support the decision whether or not to start ULT in gout patients in a faceto-face clinical setting and to agree which ULT would be preferred. Initial specifications of the content required for a decision (eg, type of ULT) and format of the DA were discussed within the steering group.

\section{Phase 2: design}

IPDAS certification criteria for DA

The IPDAS collaboration states a DA needs to present information on the decision at stake, the health condition including risk factors, the available options (first and second line) and positive (benefits) as well as negative (harms) features of each option. ${ }^{26}$ Furthermore, the DA has to offer structured guidance on deliberation which option to select.

\section{Needs assessment: patients and physicians}

A nominal group technique (NGT) was chosen to understand which characteristics/attributes of ULT are relevant for patients and should be included in the DA. The NGT facilitates quick agreement on the relative importance of an issue (in this case, the attributes of ULT) ${ }^{27}{ }^{28}$ Patients were recruited in the outpatient clinic of a regional and university hospital. All participants provided written informed consent. The discussion was facilitated by a rheumatologist and audio-recorded. Following an introduction on DAs, the purpose procedures of the meeting, participants were presented potential attributes of ULT, as obtained from a literature search preceding the NGT meetings ${ }^{29}$ Next, patients were asked to rank individually the attributes by importance from 1 (most important) to 10 (least important) on a worksheet. Patients also had the opportunity to add missing attribute(s). The individual ranks were summed across patients to obtain a ranking order. Using the initial sum scores of each attribute, a group discussion was stimulated on the initial scores. Eventually, patients were asked to rerank to support a final decision on selection of attributes to be included.

Ensuing the NGT, participants were further invited to specify the content of the attributes of ULT. For example, 'efficacy' of ULT is a key attribute, but can be specified as effect on number of gout flares, sUA and/or tophi reduction, cardiovascular risks or a combination of those. Finally, participants discussed general aspects of content and layout, including benefits and harms of the planned DA. Discussions were transcribed verbatim and content was used when developing the different parts of the prototype.

To reveal potential discrepancy between patients' and physicians' views, which attributes of ULT should be included in the DA, the ranking exercise was also carried out among rheumatologists who were not part of the steering group.

\section{Literature search}

Two literature searches were performed. The first concerned identification of potential attributes of ULT and aimed to inform the NGT meetings. A nonsystematic search was performed in PubMed for literature on DAs to support decisions on initiating a drug in a chronic disease. Keywords concerned 'chronic diseases', 'decision aid' and 'treatment'. Drug attributes were extracted and summarised into domains (eg, side effects) and specifications (eg, type of side effects, severity of side effects and frequency of side effects). The second search aimed to find data on effect of gout and its treatment on outcomes selected by patients as relevant for a decision. ${ }^{30-32}$ Using a hierarchical approach, evidence from systematic literature reviews (SLRs) of randomised controlled trials (RCTs) in patients with gout comparing the effect of ULT to placebo (outcome with opposed to without ULT) or other ULT (comparative effectiveness) would be considered as best evidence. In case of absence of SLRs or eligible RCTs, observational studies would be used. Searches and data extraction were performed by the junior researcher (RtK), and data extraction was checked by a senior researcher $(\mathrm{CvD})$. 


\section{Phase 3: development of prototype}

Based on preceding qualitative and quantitative steps (needs formulated by experts, results NGT meetings including needs among patients and literature searches), recommendations on development of DAs and expectations on outcome of SDM interventions, a prototype was developed. ${ }^{22} 3334$ During the process, members of the steering group were regularly consulted regarding the content and selection of attributes to include within the DA. For design of the DA, expertise of a design academy was consulted. To ensure readability across literacy levels, text was tested against language level (B1).

\section{Phase 4: pilot testing}

Design and participants

Pilot testing consisted of individual cognitive debriefing interviews followed by questions assessing usability of the DA. Patients were recruited in the outpatient clinic of a regional and university hospital. Patients should not have taken part in the NGT meetings and were further eligible if they were $\geq 18$ years, proficient in the Dutch language, diagnosed with gout and currently using ULT. Interviews were conducted at the outpatient clinic. All participants provided written informed consent.

\section{Cognitive debriefing}

Participants were instructed to read aloud each page of the DA in the presence of the researcher and verbalise any comments, thoughts or difficulties regarding wording, clarity, completeness of the information, visualisation, navigation through the programme and content being actionable. The researcher took field notes and prompted questions seeking clarifying comments and observations about the DA. The cognitive debriefing was audio recorded, transcribed verbatim, anonymised and analysed. The comments of the participants were summarised around the main elements of the prototype of the DA. Based on the remarks, revisions were made and the adapted prototype was tested with other participants.

\section{Usability questions}

Following the cognitive debriefing, participants completed 10 questions, adapted from the Ottawa acceptability tool, to assess the comprehensibility and usability. ${ }^{35}$ Eight closed ended items address respondents' perceptions about the DA (eg, information $(n=3$ items), design ( $\mathrm{n}=2$ items), usefulness for decisionmaking ( $\mathrm{n}=3$ items)) with Likert-scale response options (varying between 2 and 4 options) and two open-ended questions asking for potential improvements (online supplemental table S1).

\section{RESULTS}

Phase 1: specification of the scope

The scope of the DA was to facilitate a shared decision whether or not to (re)start ULT in a clinical encounter between a physician and a gout patient who has an indication to (re)start ULT. In line with the SDM paradigm, a patient can decline participation in the SDM process and rely on the physicians' decision. To enhance usability in daily practice, the content of the DA was aligned to the national and international recommendations. On that line, experts agreed that lifestyle changes should be positioned as integral part of gout management and flare prophylaxis before initiating ULT should be emphasised. Also, the potential of sUA target in management should be mentioned, and a distinction should be made between first-line and second-line ULT (first-line allopurinol and second-line febuxostat and benzbromaron). Rasburicase was not included as it is not registered for chronic urate lowering. Lesinurad was finally not included as by the time of the pilot testing, the European Medicines Agency withdrawn lesinurad on request of the market. Within these boundaries, patients can choose between available ULTs.

\section{Phase 2: design}

\section{Patient needs for information on ULT attributes}

Ten potentially important attributes of ULT (table 1) were selected from high-quality DAs retrieved from the literature search and were used in the NGT meetings. Four NGT meetings were organised within total 20 gout patients. Patients were $60 \pm 12$ years old, $18(90 \%)$ were men, disease duration was $4.1 \pm 4.9$ years and $18(90 \%))$ used currently ULT. Of note, one patient did not participate in the ranking experiment, as he trusted his physician to make the best choice for him personally.

Table 1 presents the result of the initial and final ranking. Based on a gap in the final sum score, it was agreed to include the six highest ranked attributes (effectiveness, side effects, interactions with concomitant medications, biological mechanism of action, frequency of administration and requirement for combination ULT therapy) in the DA. Patients agreed effectiveness to be the most important goal of a treatment:

'Actually, as a patient, you always want that the complaints for which you visit the doctor to stop. That can be pain or other complaints. Then you are satisfied and afterwards you will probably continue to look at whether it is harmful to your body or side effects and costs for people and yourself'.

However, when using daily ULT for long periods of time, patients felt strongly the drug should be safe and have no interactions with concomitant medications:

'Imagine I get something prescribed now but I also take something else that can clash. Does that work together? So I think that's pretty important'.

When continuing the discussion of the specific information on effectiveness (attribute ranked first), patients revealed both sUA target achievement and gout flare recurrence as most important as these were applicable to all patients. For side effects, it was preferred to be informed about the type of side effects in relation to the frequency. Patients also expressed the need for general gout information (eg, causes and risk factors) and information about lifestyle and need for flare prophylaxis. 
Table 1 Initial and final sum score and ranking by patients and final ranking by rheumatologists of the attributes during the nominal group technique meetings (order of attributes according to patients' final rank)

\begin{tabular}{|c|c|c|c|c|c|}
\hline \multirow[b]{2}{*}{ Attributes } & \multicolumn{4}{|l|}{ Patients $(n=19)$} & \multirow{2}{*}{$\begin{array}{l}\text { Rheumatologists }(n=5) \\
\text { Rank }\end{array}$} \\
\hline & Initial sum score* & Initial rank & Final sum score* & Final rank & \\
\hline Effectiveness & 26 & 1 & 26 & 1 & 1 \\
\hline Side effects & 53 & 2 & 53 & 2 & 2 \\
\hline $\begin{array}{l}\text { Interactions with } \\
\text { concomitant medications }\end{array}$ & 77 & 3 & 78 & 3 & 6 \\
\hline $\begin{array}{l}\text { Biological mechanism of } \\
\text { action }\end{array}$ & 96 & 4 & $93+$ & 4 & 4 \\
\hline $\begin{array}{l}\text { Combination therapy } \\
\text { required }\end{array}$ & 101 & 6 & $93 \dagger$ & 5 & 5 \\
\hline $\begin{array}{l}\text { Frequency of } \\
\text { administration }\end{array}$ & 97 & 5 & 98 & 6 & 3 \\
\hline Out-of-pocket cost & 137 & 7 & 141 & 7 & 7 \\
\hline Time on market & 146 & 8 & 146 & 8 & 8 \\
\hline $\begin{array}{l}\text { Branded or generic } \\
\text { specification }\end{array}$ & 151 & 9 & 155 & 9 & 9 \\
\hline Cost for the society & 161 & 10 & 162 & 10 & 10 \\
\hline
\end{tabular}

*Sum score of the ranks provides to the patients across all nominal group technique meetings in which '1' indicate the most important attribute.

†Equal final sum score by patients, but biological mechanism of action was more often ( $36 \%$ vs $11 \%)$ prioritised in the top three as attribute.

Finally, patients preferred the visualisation of risk communication on the most important outcome as icon arrays.

When comparing the rank order of attributes between patients and rheumatologist, the latter had underestimated the importance for patients of potential interactions of ULT with other drugs.

\section{Literature search}

Three SLRs were identified, synthetising efficacy of allopurinol, febuxostat and uricosurics. ${ }^{30-32}$ As the last search of each review was older than 12 months, updates were conducted using each SLR's original search strategy. Overall, six RCTs were selected comparing effect of ULT to placebo or other ULTs on flares as outcome (the preferred outcome for patients) ${ }^{36-41}$ After tabulating the study characteristics, data turned out to be too heterogeneous (eg, exclusion criteria, definition of flares, sUA level at inclusion) and follow-up time was too short to provide meaningful data on efficacy on flares (as flares provoked by ULT initiation distorts long-term efficacy). Therefore, an available review of five longitudinal studies reporting data on the association of flares according to sUA category was used. ${ }^{42}$ The study best fitting our target population, concerned patients with rheumatologists diagnosed gout and clinically confirmed flares. ${ }^{43}$ Of note, the majority of patients were (not yet) treated with ULT during follow-up. As the relation between sUA and flare was steep, it was decided to distinguish two sUA categories, differing in future flares risk. In patients with an sUA of $>0.36-<0.55 \mathrm{mmol} / \mathrm{L}$, future flares risk was $48 / 100$ and in patients with a $\mathrm{sUA} \geq 0.55 \mathrm{mmol} / \mathrm{L}$ future flare risk was $90 / 100$ within a follow-up period of at least 1 year. Patients who would reach the sUA target $(\leq 0.36 \mathrm{mmol} / \mathrm{L})$ would have a future flares risk of $12 / 100$. To understand the relative efficacy of the different ULTs, one head-to-head RCT comparing allopurinol, febuxostat and placebo concluded a stronger impact of febuxostat compared with allopurinol on sUA but not on flares. ${ }^{30} 36$

\section{Phase 3: development of prototype}

A six-page paper DA, personalised according to the patient's current sUA level, being $>0.36$ but $<0.55 \mathrm{mmol} / \mathrm{L}$ or $\geq 0.55 \mathrm{mmol} / \mathrm{L}$, was developed (see table 2 for sources of content). The first page explicitly stated the decision that had to be taken, described the health condition and explored the (personalised) risk factors (eg, comorbidities, gender, tophi, sUA) for gout, and previous ULT (and potential side effects) use. Page two visualised the (personalised) risk on future gout flares without ULT by icons arrays of a gout flare in the first metatarsophalangeal joint (first MTP). Page three described the role of lifestyle changes, comprising weight loss if obese and diminution of alcohol consumption if present and the treatment of flares. Page four introduced the benefits of ULT on the risk of future flares when reaching a sUA target $(\leq 0.36 \mathrm{mmol} / \mathrm{L})$, again visualised by icons arrays. The chance of sUA target achievement when initiating ULT was added as text below the icon arrays. Additionally, the recommendation to lower sUA $\leq 0.30 \mathrm{mmol} / \mathrm{L}$ in tophaceous gout, and the need for flare prophylaxis on initiation of ULT was emphasised. An option grid (page five) provided an overview of the attributes selected by patients and the steering group distinguishing the available first-line and second-line ULT options. The attribute 
Table 2 Information sources used for the various sections of the decision aid

\begin{tabular}{ll}
\hline Sections of prototype & Information sources \\
\hline $\begin{array}{l}\text { I: Health condition and personal risk } \\
\text { factors }\end{array}$ & Landmark gout literature provided by the steering group. \\
II: The personalised risk on future gout & Literature search of Cochrane database did not meet the needs; a review on the \\
flares without ULT & relation between sUA and gout flares was used instead. \\
III: Lifestyle changes and treatment of & SLR on effectiveness and side effect treatment acute gout flares. \\
acute flares & SLR on effect lifestyle in gout outcomes provided by the steering group. \\
& National and international recommendations on gout management. \\
& Dutch exercise norm. \\
IV: Effect of ULT & Literature search of Cochrane database did not meet the needs; a review on the \\
& relation between sUA and gout flares was used instead. \\
V: Option grid & Role of flare prophylaxis added per advice of the steering group. \\
& Literature search on available decision aids provided a list of attributes that \\
& informed the NGT meetings. \\
& NGT ranked attributes (six highest were included). \\
& Cochrane review on comparative effectiveness of ULT on gout flares. \\
& National and international recommendations on gout management. \\
& For side effects information and data provided by the Dutch Healthcare Institute \\
(www.farmacotherapeutischkompas.nl).
\end{tabular}

NGT, nominal group technique; SLR, systematic literature review; sUA, serum uric acid; ULT, urate-lowering therapy.

combination ULT required was removed, as lesinurad had not received reimbursement in the Netherlands, and none of the other ULT required combination with another ULT. The last page asked patients whether they have remaining unanswered questions and offered them the opportunity to discuss personal views, worries and believes about gout and gout treatment. Finally, patients were invited to make a decision, whether or not to start ULT and to consent with the chosen ULT option. Of note, the same DA can be used for patients starting or restarting ULT, as only the ULT options available according to the healthcare professional in the option grid might differ (eg, change from one ULT to another in case of previous side effects). Clearly, our DA is a professional administered tool and healthcare professionals usually require some level of training/experience to apply the tool.

\section{Phase 4: pilot testing}

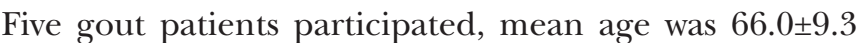
years, all were men, average disease duration was $16.4 \pm 15.1$ years, educational level was high $(n=2)$, intermediate $(n=2)$ and low $(n=1)$, and all used currently ULT.

\section{Cognitive debriefing}

Overall, patients appreciated the provision of valuable information of different ULT options and attributes:

'Very clear and informative. If I had seen this DA earlier, I would have started with ULT sooner now that I have seen all the pros and cons'

Minor improvements were suggested for every page on language (eg, replacing words, shortening or rephrasing of sentences), font and symbols (table 3). Patients confirmed that the general gout information and lifestyle information were useful and necessary in obtaining a complete picture of gout:

'Using your ULT tablets is very important, yet it is always additional to lifestyle. You should always eat healthy and have enough daily activity'.

Notwithstanding, one patient suggested to provide more practical advices on healthy lifestyle, for example, maximum units of alcohol consumptions and this information was added to the DA.

Finally, patients pointed to the large amount of information and potential cognitive burden, and recommended to take the DA home after consultation. For this reason, also the telephone and email address of the gout clinic were included in the DA in case further questions would raise.

\section{Usability questions}

Patients appreciated the information, design and usefulness for SDM (online supplemental table S1). The openended questions revealed no new information compared with the cognitive debriefing.

\section{DISCUSSION}

A systematic process was followed to develop a DA to facilitate a shared decision whether or not to (re)start ULT in gout patients with an indication to initiate ULT. Patients and physicians were involved in the design, prototype development and pilot testing of the DA. Overall, participants found the DA valuable to facilitate the treatment decision, optimise communication and increase patient empowerment. In line with SDM principles, a first question when engaging in SDM is whether a patient wants 
Table 3 Comments by patients in the pilot test on every page of the decision aid and adaptations made by the steering group

\begin{tabular}{ll}
\hline Main element & Comments \\
\hline $\begin{array}{l}\text { I: Health condition and personal risk } \\
\text { factors }\end{array}$ & $\begin{array}{l}\text { Gout flares can occur in more body parts } \\
\text { than the big toe, for example in the ears } \\
\text { Gout can give more severe problems than } \\
\text { only flares and pain. Elaborate on other } \\
\text { severe problems } \\
\text { Some risk factors are not applicable for } \\
\text { individual patients }\end{array}$ \\
II: The personalised risk on future & $\begin{array}{l}\text { Not clear that the icon arrays are gout flares } \\
\text { in the first metatarsophalangeal joint }\end{array}$ \\
gout flares without ULT & $\begin{array}{l}\text { Not clear if the risk of future gout flares was } \\
\text { independent of the personal sUA level }\end{array}$
\end{tabular}

\begin{tabular}{|c|c|c|}
\hline \multirow[t]{2}{*}{$\begin{array}{l}\text { III: Lifestyle changes and treatment } \\
\text { of acute flares }\end{array}$} & $\begin{array}{l}\text { Give more detailed information for advices } \\
\text { related to proportions (eg, drinks or red } \\
\text { meat) }\end{array}$ & Detailed for alcohol consumption \\
\hline & Specify the non-citrus fruits with examples & Overruled/rejected by the steering group \\
\hline \multirow[t]{2}{*}{ IV: Effect of ULT } & Mention the specific sUA target of ULT & Added the sUA target of $\leq 0.36 \mathrm{mmol} / \mathrm{L}$ \\
\hline & $\begin{array}{l}\text { Mention number of future flares that will } \\
\text { occur within the period considered in the } \\
\text { figure with icon arrays }\end{array}$ & $\begin{array}{l}\text { Added icon arrays presenting number of } \\
\text { patients with at least one gout flare }\end{array}$ \\
\hline \multirow[t]{3}{*}{ V: Option grid } & $\begin{array}{l}\text { Mention here also the generic name of ULTs. } \\
\text { A patient that used desuric, did not know } \\
\text { this was also called benzbromaron }\end{array}$ & $\begin{array}{l}\text { Generic names were added to the option } \\
\text { grid for all the ULTs }\end{array}$ \\
\hline & Increase font style within the option grid & Font of symbols and text was increased \\
\hline & Add rasburicase & $\begin{array}{l}\text { The DA clarifies only ULTs for long-term } \\
\text { control of sUA are included }\end{array}$ \\
\hline
\end{tabular}

DA, decision aid; sUA, serum uric acid; ULT, urate-lowering therapy.

to be involved or prefers that the physician makes the decision whether or not to (re)start ULT. Among the 25 patients providing input for the DA, only one patient preferred the physician to make the final treatment decision. Patients appreciated the option grid, including the information on alternative options when the initial choice option would fail. Notwithstanding, patients also recognised the intellectual burden and recommended to take home the DA. On this line, we chose to create a paper-based DA (opposed to web-based) to facilitate a face-to-face clinical setting. In a COVID-19 era with remote visits, a web-based version that remains accessible to patients may be more appropriate. A study in the USA on rheumatologists' views and practices related to SDM in gout treatment revealed that $70 \%$ of the rheumatologists reported to offer patients offering a choice whether or not to start ULT without a DA. ${ }^{44}$ Our DA might support patients and physicians in this shared decision.

To enhance usability of a DA in daily practice, it is essential to adhere to national and/or international recommendations (EULAR/ACR). The distinction in the current DA between the first-line (allopurinol) and second-line

\section{Adaptations made}

Other commonly involved joints added to the text

Tophi in skin and bone explicitly mentioned

The risk factor personalised by adding tick boxes

Specifically added that the most common gout flare location was illustrated

The DA was personalised for patients with an initial sUA level between (>0.36$<0.55 \mathrm{mmol} / \mathrm{L}$ and $\geq 0.55 \mathrm{mmol} / \mathrm{L}$ ).

\section{Detailed for alcohol consumption}

Overruled/rejected by the steering group Added the sUA target of $\leq 0.36 \mathrm{mmol} / \mathrm{L}$ Added icon arrays presenting number of patients with at least one gout flare

Generic names were added to the option Font of symbols and text was increased The DA clarifies only ULTs for long-term control of sUA are included mentioned (eg, time and date) 
dose, this does not translate into better effectiveness on flare reduction. Yet, the relationship between sUA and gout flares is complex.

In the literature, only one DA prototype was designed in an Asian community for gout treatment. ${ }^{46}$ However, information on flares risk with and without ULT was lacking. Notwithstanding, this is a key aspect of a DA. This lack of state of the art development of current DAs highlights the value of our stepwise and transparent description of the development process of our DA.

Some limitations should be recognised. Due to practical circumstances, among which consequences of COVID-19 restrictions, only five patients were included in the pilot test. As a consequence, feedback might have been homogenous. Therefore, when further testing effectiveness of the DA-specific needs of patients who are ULT naïve or with lower health literacy should receive attention. Fortunately, our DA is flexible for adaptation to new or personalised evidence on (treatment of) gout or needs of patients. Appropriate testing of the (cost)-effectiveness of the DA will require, a (semi) experimental trial. Consistent with the paradigm of patient-centred care, Outcome Measures in Rheumatology reached consensus that not only adherence to the chosen outcome is an important core outcome but also ${ }^{1}$ knowledge of options, their potential benefits and harms'; chosen option aligned with each patient's values and preferences ${ }^{3}$; confidence in the chosen option ${ }^{4}$; satisfaction with the decisionmaking process $a^{5} d^{5}$ potential negative consequences (eg, time and costs). Implementation of SDM and the use of a DA in clinical practices require changes in patientphysician communication. Even before evidence on the effectiveness on different outcomes in various subgroups will be available, our DA can be used to gain experience with SDM in the context of patient-centred care.

\section{CONCLUSION}

We systematically developed and pilot tested a DA to (re)start with ULT. This study provides initial support for usability of a DA for gout patients eligible to start or restarting ULT. Testing of effectiveness on gout outcome and patient experiences in clinical practice is a necessary step.

Acknowledgements We thank Sophie Nelissen and Gaston Jamin for their expertise in the design process and during the development of the DA. We also would thank all the gout patients for their time and comments during the interviews and the pilot testing of the DA.

Contributors RtK analyzed and interpreted the patient data regarding the usability of the decision aid. RtK, $A B$, and CvD contributed most to the development of the decision aid itself. The content of the decision aid was agreed upon the contribution of RtK, TJ, MJ, HdV, CvD, and $A B$. AB was major contributor in writing the manuscript. All authors read and approved the final manuscript.

Funding This work was supported by the MUMC+/VieCuri Science Funds.

Competing interests None declared.

Patient consent for publication Not applicable.

Ethics approval This study involves human participants and was approved by Ethical Committee of Maastricht University Medical Center (METC: 2018-0801). Participants gave informed consent to participate in the study before taking part.
Provenance and peer review Not commissioned; externally peer reviewed.

Data availability statement All data relevant to the study are included in the article or uploaded as supplementary information. All data generated or analysed during this study are included in this published article and its supplementary information) files.

Open access This is an open access article distributed in accordance with the Creative Commons Attribution Non Commercial (CC BY-NC 4.0) license, which permits others to distribute, remix, adapt, build upon this work non-commercially, and license their derivative works on different terms, provided the original work is properly cited, appropriate credit is given, any changes made indicated, and the use is non-commercial. See: http://creativecommons.org/licenses/by-nc/4.0/.

\section{ORCID iDs}

Ritch te Kampe http://orcid.org/0000-0002-3409-1444

Annelies Boonen http://orcid.org/0000-0003-0682-9533

\section{REFERENCES}

1 Dalbeth N, Merriman TR, Stamp LK. Gout. Lancet 2016;388:2039-52.

2 Nielsen SM, Bartels EM, Henriksen M, et al. Weight loss for overweight and obese individuals with gout: a systematic review of longitudinal studies. Ann Rheum Dis 2017;76:1870-82.

3 Choi HK, McCormick N, Lu N, et al. Population impact attributable to modifiable risk factors for hyperuricemia. Arthritis Rheumatol 2020;72:157-65.

4 FitzGerald JD, Dalbeth N, Mikuls T, et al. 2020 American College of rheumatology guideline for the management of gout. Arthritis Care Res 2020;72:744-60.

5 Pillinger $\mathrm{MH}$, Mandell BF. Therapeutic approaches in the treatment of gout. Semin Arthritis Rheum 2020;50:S24-30.

6 Pascart T, Lioté F. Gout: state of the art after a decade of developments. Rheumatology 2019;58:27-44.

7 Doghramji PP, Fermer S, Wood R, et al. Management of gout in the real world: current practice versus guideline recommendations. Postgrad Med 2016;128:106-14.

8 Conway R, Coughlan RJ, Carey JJ. Adherence to uric acid treatment guidelines in a rheumatology clinic. Clin Rheumatol 2012;31:1707-11.

9 Roddy E, Packham J, Obrenovic K, et al. Management of gout by UK rheumatologists: a British Society for rheumatology national audit. Rheumatology 2018:57:826-30.

10 Janssen CA, Jansen TLTA, Oude Voshaar MAH, et al. Quality of care in gout: a clinical audit on treating to the target with urate lowering therapy in real-world gout patients. Rheumatol Int 2017;37:1435-40.

11 Rai SK, Choi HK, Choi SHJ, et al. Key barriers to gout care: a systematic review and thematic synthesis of qualitative studies. Rheumatology 2018;57:1282-92.

12 Spaetgens B, Pustjens T, Scheepers LEJM, et al. Knowledge, illness perceptions and stated clinical practice behaviour in management of gout: a mixed methods study in general practice. Clin Rheumatol 2016;35:2053-61.

13 Aung T, Myung G, FitzGerald JD. Treatment approaches and adherence to urate-lowering therapy for patients with gout. Patient Prefer Adherence 2017;11:795-800.

14 van Onna $\mathrm{M}$, Hinsenveld $\mathrm{E}$, de Vries $\mathrm{H}$, et al. Health literacy in patients dealing with gout: a qualitative study. Clin Rheumatol 2015;34:1599-603.

15 Gill I, Dalbeth N, 'Ofanoa M, et al. Interventions to improve uptake of urate-lowering therapy in patients with gout: a systematic review. BJGP Open 2020;4:bjgpopen20X101051.

16 Leavitt M, Wolfe A. Medscape's response to the Institute of medicine report: crossing the quality chasm: a new health system for the $21 \mathrm{st}$ century. MedGenMed 2001;3:233-5.

17 Barratt A, Trevena L, Davey HM, et al. Use of decision AIDS to support informed choices about screening. BMJ 2004;329:507-10.

18 Elwyn G, O'Connor A, Stacey D, et al. Developing a quality criteria framework for patient decision AIDS: online international Delphi consensus process. BMJ 2006;333:417.

19 O'Connor AM, Llewellyn-Thomas HA, Flood AB. Modifying unwarranted variations in health care: shared decision making using patient decision AIDS. Health Aff 2004;Variation:VAR-63-VAR-72.

20 Härter M, Moumjid N, Cornuz J, et al. Shared decision making in 2017: international accomplishments in policy, research and implementation. Z Evid Fortbild Qual Gesundhwes 2017;123124:1-5.

21 Barry MJ, Edgman-Levitan S. Shared decision making--pinnacle of patient-centered care. N Engl J Med 2012;366:780-1. 
22 Stacey D, Légaré F, Lewis K, et al. Decision AIDS for people facing health treatment or screening decisions. Cochrane Database Syst Rev 2017;4:CD001431-CD

23 Richette P, Doherty M, Pascual E, et al. 2016 updated EULAR evidence-based recommendations for the management of gout. Ann Rheum Dis 2017;76:29-42.

24 FitzGerald JD, Dalbeth N, Mikuls T, et al. 2020 American College of rheumatology guideline for the management of gout. Arthritis Care Res 2020;72:744-60.

25 Coulter A, Stilwell D, Kryworuchko J, et al. A systematic development process for patient decision AIDS. BMC Med Inform Decis Mak 2013;13 Suppl 2:S2.

26 Joseph-Williams N, Newcombe R, Politi M, et al. Toward minimum standards for certifying patient decision AIDS: a modified Delphi consensus process. Med Decis Making 2014;34:699-710

27 Delbecq AL, Van de Ven AH, Gustafson DH. Group techniques for program planning: a guide to nominal group and Delphi processes: Scott, Foresman, 1975.

28 Hiligsmann M, van Durme C, Geusens $\mathrm{P}$, et al. Nominal group technique to select attributes for discrete choice experiments: an example for drug treatment choice in osteoporosis. Patient Prefer Adherence 2013;7:133-9.

29 McMillan SS, King M, Tully MP. How to use the nominal group and Delphi techniques. Int J Clin Pharm 2016;38:655-62.

30 Seth R, Kydd ASR, Buchbinder R, et al. Allopurinol for chronic gout. Cochrane Database Syst Rev 2014;353.

31 Kydd ASR, Seth R, Buchbinder R, et al. Uricosuric medications for chronic gout. Cochrane Database Syst Rev 2014;2014:CD010457.

32 Tayar JH, Lopez-Olivo MA, Suarez-Almazor ME, et al. Febuxostat for treating chronic gout. Cochrane Database Syst Rev 2012;353:CD008653.

33 O'Connor AM, Légaré F, Stacey D. Risk communication in practice: the contribution of decision AIDS. BMJ 2003;327:736-40.

34 Toupin-April K, Barton JL, Fraenkel L, et al. OMERACT development of a core domain set of outcomes for shared decision-making interventions. J Rheumatol 2019;46:1409-14.

35 O'Connor AM, Drake ER, Fiset V, et al. The Ottawa patient decision AIDS. Eff Clin Pract 1999;2:163-70.
36 Schumacher HR, Becker MA, Wortmann RL, et al. Effects of febuxostat versus allopurinol and placebo in reducing serum urate in subjects with hyperuricemia and gout: a 28-week, phase III, randomized, double-blind, parallel-group trial. Arthritis Rheum 2008;59:1540-8.

37 Taylor TH, Mecchella JN, Larson RJ, et al. Initiation of allopurinol at first medical contact for acute attacks of gout: a randomized clinical trial. Am J Med 2012;125:1126-34.

38 Tausche A-K, Alten R, Dalbeth N, et al. Lesinurad monotherapy in gout patients intolerant to a xanthine oxidase inhibitor: a 6 month phase 3 clinical trial and extension study. Rheumatology 2017;56:2170-8.

39 Dalbeth N, Saag KG, Palmer WE, et al. Effects of febuxostat in early gout: a randomized, double-blind, placebo-controlled study. Arthritis Rheumatol 2017:69:2386-95.

40 Saag KG, Becker MA, Whelton A, et al. Efficacy and safety of febuxostat extended and immediate release in patients with gout and renal impairment: a phase III placebo-controlled study. Arthritis Rheumatol 2019;71:143-53.

41 Sun R, Lu J, Li H, et al. Evaluation of febuxostat initiation during an acute gout attack: a prospective, randomized clinical trial. Joint Bone Spine 2020;87:461-6.

42 Shiozawa A, Szabo SM, Bolzani A, et al. Serum uric acid and the risk of incident and recurrent gout: a systematic review. J Rheumatol 2017;44:388-96.

43 Shoji A, Yamanaka H, Kamatani N. A retrospective study of the relationship between serum urate level and recurrent attacks of gouty arthritis: evidence for reduction of recurrent gouty arthritis with antihyperuricemic therapy. Arthritis Rheum 2004;51:321-5.

44 Singh JA, Richards JS, Chang E, et al. Shared decision-making in gout treatment: a national study of rheumatology provider opinion and practice. Clin Rheumatol 2021;40:693-700.

45 Trevena LJ, Zikmund-Fisher BJ, Edwards A, et al. Presenting quantitative information about decision outcomes: a risk communication primer for patient decision aid developers. BMC Med Inform Decis Mak 2013;13 Suppl 2:S7.

46 Meyappan M, Loh WSA, Tan LY, et al. Development of a novel gout treatment patient decision aid by patient and physician: a qualitative research study. Health Expect 2021;24:431-43. 